\title{
Factors Influencing an Organisation's Intention to Adopt Cloud Computing in Saudi Arabia
}

\author{
Nouf Alkhater \\ School of Electronics and Computer Science \\ University of Southampton \\ Southampton, United Kingdom \\ nrma1c12@ecs.soton.ac.uk
}

\author{
Gary Wills \\ School of Electronics and Computer Science \\ University of Southampton \\ Southampton, United Kingdom \\ gbw@ecs.soton.ac.uk
}

\author{
Robert Walters \\ School of Electronics and Computer Science \\ University of Southampton \\ Southampton, United Kingdom \\ rjw1@ecs.soton.ac.uk
}

\begin{abstract}
Cloud computing is paradigm that has emerged to deliver IT services to consumers as a utility service over the Internet. In developing countries, particularly Saudi Arabia, cloud computing is still not widely adopted. As a result, this study seeks to investigate the most influential factors that can encourage an organisation to use the cloud or which might impede them from moving to it. This research proposes an integrated model that incorporates aspects of the TechnologyOrganisation-Environment (TOE) framework and integrates the critical factors from existing theories along with other factors to examine the impact of this variable on the adoption decision of enterprises. Future work will be focused on confirming the proposed model.
\end{abstract}

Keywords- cloud computing; adoption; technological factors; organisational factors; environmental factors

\section{INTRODUCTION}

Cloud computing is paradigm which combines several existing IT technologies into one service. Most of the IT technologies that are being used in cloud computing are already being used individually, such as virtualisation and Web 2.0, but in cloud computing some of their capabilities are selected to create the cloud environment [1]. Cloud computing was a dream in the sixties [2], when John McCarthy predicted of the power of computing to provide utility services. In order to achieve this goal, a number of paradigms have been developed such as grid computing, but none succeeded in offering a public service the way cloud computing has.

Cloud computing offers several benefits for enterprises. The cloud frees organisations from having to set up an IT infrastructure and allows them to rent resources and pay only for the services they use [3]. This can reduce costs and save a lot of money for both small and large enterprises. In addition, cloud computing increases flexibility and offers an attractive opportunity for enterprises to grow [4]. However, some organisations still concerns about the idea of shifting a present system to the cloud.

A number of researchers in the past have made several attempts to help decision makers in the organisation to address their concerns about cloud adoption. It is noticeable that most of these proposed frameworks and models are focused on identifying the costs and benefits of adopting cloud computing and only a few empirical studies examine the influential factors on adoption decision of cloud computing at an organisation level such as [5],[6]. Furthermore, the adoption rate of cloud computing is still in the beginning stage in Saudi Arabia. Therefore, this study aims to investigate these influential factors in depth and understand why organisations are willing to move to the cloud or why not. This research proposed an integrated model in an attempt to find out what might encourage an organisation to use cloud services or might impede them from using it.

The structure of the paper is as it follows. Section II discusses the key benefits that drive organisations to adopt cloud and existing theories used to measure acceptance of technologies, as well as provides a critical review of other proposed models based on these theories. Section III presents the proposed model for cloud computing adoption. Section IV provides an overview of the research methodology. The preliminary results are presented in Section V. Finally, conclusion and future work in Section VI.

\section{LITERATURE REVIEW}

\section{A. Benefits of Cloud Adoption}

There are several benefits and advantages of adopting cloud computing for organisations. Firstly, the cloud can reduce costs and save money for both small and large enterprises because it offers an outsourcing model which allows them to rent resources and pay only for the service that they use, rather than building up in-house IT infrastructure. Moreover, the maintenance of IT resources and the upgrades are managed by a third party, which allows organisations to transfer responsibility and save money [1],[3],[7].

Another advantage of cloud migration is that it enables enterprises to grow and scale their services easily without delay and according to their specific needs. For example, sales staff can provide new products and sell them easily and quickly in the cloud environment. Furthermore, by using a cloud environment in organisations, the numbers of IT infrastructure resources, such as servers, will be minimised, and consequently the cost of energy consumption will be reduced [4],[8]. In addition, cloud computing allows the enterprises to run their applications 
with a minimum failure rate. Finally, the flexibility of delivering computing services is the key benefit that drives organisations to migrate their IT systems to the cloud [9].

\section{B. Technology Adoption Theories}

There are several models and theories proposed by researches to measure the acceptance of a new technology. This section starts with illustrating the three main models (Technology-Organisation-Environment (TOE) framework, the Diffusion of Innovations (DOI) theory and the institutional theory) that have been widely used by researchers to analyse the adoption of IT technology.

\section{- Technology-Organisation-Environment Framework}

The TOE framework was proposed by Tornatzky and Fleischer [10], to analyse the adoption of new IT technologies at an organisational level. This framework investigated the impact of three factors (Technology, Organisation and Environment) on the organisation's decision to adopt a new technology. The technology aspect describes the effect of internal and external technologies of the firm and how adopting new technology can influence the firm [10],[11]. The organisational context refers to different measures of the organisation. For example, firm size, scope and complexity of managerial structure. These measures have a significant impact on the adoption decision. Lastly, the environmental context is the field where an organisation runs its business; the industry, competitors and government regulation define the environmental context.

\section{- Diffusion of Innovation Theory}

The DOI theory was developed by Rogers [12]. DOI is a widely used model to explain why and how adoption of new ideas and technologies occurs at individual and organisational levels. The DOI theory posits that there are five technological attributes that have a direct impact on the adoption rate. These attributes are relative advantage, complexity, compatibility, trialability and observability; Rogers [12] defines these aspects as follows:

- Relative advantage: "The degree to which an innovation is perceived to be better than the idea it supersedes."

- Compatibility: "The degree to which an innovation is perceived as consistent with the existing values, past experiences, and needs of potential adopters."

- Complexity: " The degree to which an innovation is perceived as relatively difficult to understand and use."

- Trialability: "The degree to which an innovation may be experimented with on a limited basis."

- Observability: "The degree to which the results of an innovation are visible to others."
The decision to adopt technology at an organisational level is more difficult due to the dependence on different views from a number of people at the organisation. Also, the decision of an organisation to adopt technology is affected by different characteristics, such as the leader's characteristics and internal and external characteristics of the organisation. These characteristics are similar to the organisational and technological factors discussed in the TOE framework. However, the TOE framework did not focus on analysing the attributes of technology, as the DOI theory does [12],[13].

\section{- Institutional Theory}

The organisational environment is a vital factor to define institutional structure and actions as institutional theory states [14],[15]. Institutional theory is a common theory used in a number of research studies for examining IT adoption. This theory includes new and important factors in the environmental element of the TOE framework. These new factors are trading partners and competitors, which are external pressures that affect an organisation's decision to adopt a technology. The theory claims that the decision to adopt technology is not only based on internal factors but is also influenced by other factors such as community, culture and legitimacy concerns.

\section{A Review of Proposed Models for Cloud Computing Adoption}

This section discusses different published studies based on the previous theories. These studies aim to identify the factors that have had a major influence on an organisation's decision to adopt cloud computing.

Low et al. [5] proposed a model based on the TOE framework. The purpose of this study was to identify the factors and determine their impacts on the decision to adopt cloud computing in higher education in Taiwan. The data in this study was collected based on a survey of the higher education industry in Taiwan. The findings indicated that there are five factors - namely relative advantage, top management support, organisation size, competitive pressure, and trading partner pressure - which have a major influence on the decision to adopt cloud computing. A further study was conducted by Chang et al. [16] based on the TOE framework and DOI theory, aiming to investigate the factors that affect cloud computing adoption in Vietnamese companies. In their study they identified a number of factors, similar to the former study. However, their work has not provided any results because their model was in the initial stage. It is noticeable that these two studies have not considered security factors that affect an organisation's decision to adopt cloud computing, although security is the one of the main concerns for an organisation that wants to apply new technology.

Also, Nkhoma and Dang [6] developed a conceptual model using the TOE framework to examine benefits and barriers of adopting cloud computing as well as the impacts 
of the environmental and technological variables on the adoption decision. They identified a number of barriers as factors that influence the intention to use cloud computing. These barriers are security, reliability and availability, compliance with policies, IT standards, extendibility of existing applications and the extent of their compatibility with cloud use. In this study they also investigated the influence of the Adopter's Style (AS).Their results reveal that AS has a major negative impact on the decision to adopt cloud computing whereas the rest of the barriers and benefits have not shown any effect on the adoption decision. Their results are unclear, which indicates that this study needs to re-validate the proposed model and results.

Abdollahzadehgan et al. [17] analysed only the effect of organisational factors on cloud computing adoption in small and medium size enterprises (SMEs). They identified three factors (top management support, firm size and technology readiness) using a TOE framework. These factors are very helpful for organisation to assess their situations if they want to adopt cloud, especially the SMEs. However, in this study the authors focused on analysing organisational factors and omitted other factors (such as technological, environmental and security factors) that affect on organisation intent to adopt cloud.

\section{THE PROPOSED MODEL}

An integrated model has been developed to examine what are the most important factors that affect an organisation's decision to adopt the cloud. This model incorporates aspects of the TOE framework [10], also integrating the critical factors from DOI theory [12] and institutional theory [14] along with other factors (trust, privacy and physical location) that have not yet been studied but that may influence the organisation decision to adopt cloud. The research model consists of three fundamental factors: technological, organisational, and environmental factors. These are illustrated in the cloud computing adoption model (Figure 1).

\section{A. Technological Factor}

The technological factor describes the characteristics of the cloud technology and identifies the factors that affect an organisation's decision to adopt this technology.

- Availability: Availability is an influential factor in relation to an organisation's decision to adopt cloud computing. Cloud computing offers resources online, meaning that the consumer can access the cloud from anywhere and at any time. This means the system needs to function properly and must be available to use whenever it is requested.

- Reliability: The reliability of the cloud is another major factor in the decision. It refers to the ability of a system to fulfil its intended function in a proper manner as expected. Reliability involves ensuring a high quality of service to end users, with a high transmission rate, minimum rate of errors, and fast recovery.

- Security: Refers to the level of security procedures in place to protect information or the system from unauthorised access or any other security events. Lack of security is one of the biggest doubts for many organisations that intend to adopt the cloud [18].

- Privacy: Defined as confidentiality of data, where only authorised users can access it. It is the main concern for organisations thinking about cloud computing because when using a cloud service an organisation cannot fully control the information stored on cloud-based servers.

- $\quad$ Trust: Trust refers to the reliance on another entity and the belief that this entity will function as expected. Trust in the cloud environment heavily depends on trusting the service itself and the provider to provide a trusted level of authenticity, integrity and confidentiality in regard to the service and the stored data.

- Relative advantage: Relative advantage is an element of the DOI model [12]. This factor refers to the level of benefit to an organisation if they decide to move into cloud computing.

- Compatibility: Refers to the ability of the existing application to be compatible with the cloud. The compatibility of a firm's applications with the cloud environment is a real problem that an organisation needs to consider carefully when considering use of the cloud. This factor was identified in the Rogers model [12].

- Complexity: An organisation normally considers the degree of difficulty involved in using new technology as an important element in their decision before adopting this technology. This factor is also an element of the DOI model [12].

\section{B. Organisational Factor}

The organisational factor describes the characteristics of an organisation that might have a significant impact on their decision.

- Top management support: Top management play an important role and have a significant impact on the adoption rate of IT innovations at the organisational level. Support from top management is essential because they have the ability to make the change and execute acceptance of the cloud. This change in the organisation needs a supportive decision from top management.

- Organisation size: The size of the organisation is another influential factor. The organisation size is defined by the number of employees, the amount of investments, the target market and annual revenue.

- Technology readiness: The technological readiness of organisations, meaning the degree of readiness of the IT infrastructure and the human resources in terms of cloud computing.

\section{Environmental Factor}

The environmental factor determines the environmental elements that might affect an organisation's intent to use cloud technology. 
- Compliance with regulations: This is an influential factor that can make a firm reluctant to move into cloud computing. This concern comes from the fact that there are no governmental regulations or rules that can support the firm in the event of a data breach. The lack of IT standards is a real problem that might obstruct adoption decisions [4].

- Competitive pressure: Defined as the degree of pressure that an organisation faces from competitors. In a highly competitive industry, an organisation encounters pressure from competitors to adopt new technologies.This pressure forces some organisations to adopt cloud computing technology and gain a great benefit, more business facilities and better operational efficiency.

- Trading partner pressure: This is another pressure affecting the firm's adoption decision. This pressure comes from vendors or other partners who might adopt cloud computing. Consequently, this might encourage an organisation to adopt this technology.

- Physical location: The physical location is a critical factor that affect an organisation's decision to adopt cloud computing for several reasons. First there are no international policies or regulations for data protection in cloud. Second the fact that some of the cloud providers store the data in another country without disclosing this to the end users [19].

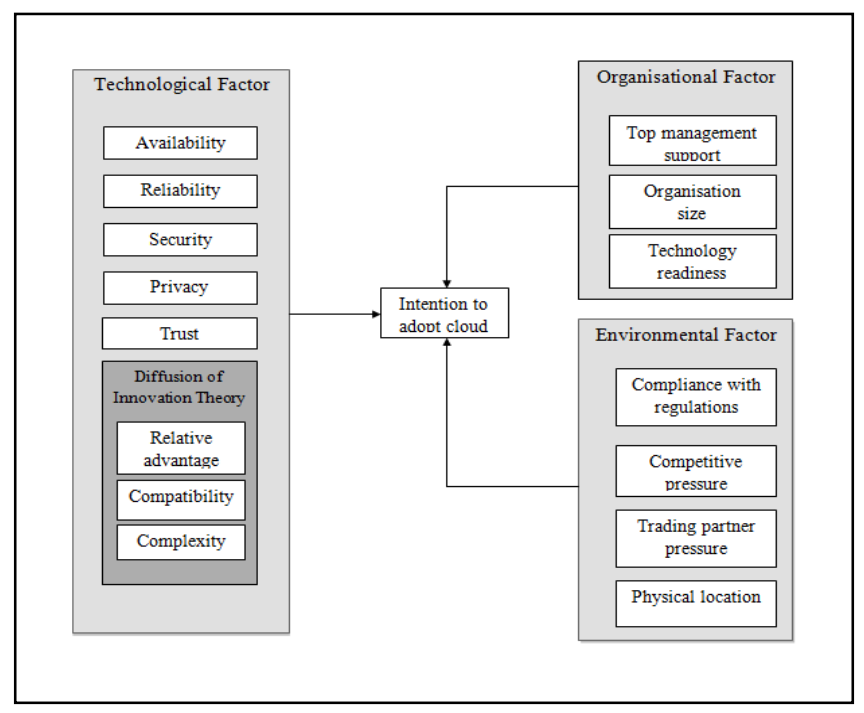

Figure 1 An integrated model for cloud computing adoption

\section{METHODOLOGY}

The data was collected using semi-structured interviews. The interviews were conducted with twenty IT experts at different organisations in Saudi Arabia. The aim of interviews was to improve the proposed model and explore other factors that were not mentioned in previous studies before carrying out a survey. All the participants were working in IT departments in different sectors, such as manufacturing, engineering and energy. All participants had at least five years' working experience, and most of the interviewees were IT managers and heads of IT department, so they had the ability to understand the current situation of their organisations and future trends. This study was conducted on a large, medium and small enterprises. Seven of these companies have already adopted cloud computing while thirteen of them have not.

\section{RESULTS}

This section presents the results of the initial study. The interviewees were asked for their opinion about all the proposed factors in Section III using closed-ended questions. The aim of the questions was to assess the importance of the proposed factors to an organisation's intention to adopt cloud services, from the experts' point of view. The collected data was analysed using SPSS software and tested using the one-sample t-test. The test value was defined as 3 from the five-point Likert scale, which ranged from 5 (very important) to 1 (not relevant). The results are presented in Table I.

Table I. Results of one-sample t-test

\begin{tabular}{|c|c|c|}
\hline Factors & p-value & Result \\
\hline Availability & $<0.001$ & Statistically significant \\
\hline Reliability & $<0.001$ & Statistically significant \\
\hline Security & $<0.001$ & Statistically significant \\
\hline Privacy & $<0.001$ & Statistically significant \\
\hline Trust & $<0.001$ & Statistically significant \\
\hline Relative advantage & $<0.001$ & Statistically significant \\
\hline Compatibility & $<0.001$ & Statistically significant \\
\hline Complexity & $<0.001$ & Statistically significant \\
\hline Top management support & $<0.001$ & Statistically significant \\
\hline Organisation size & .003 & Statistically significant \\
\hline Technology readiness & $<0.001$ & Statistically significant \\
\hline Compliance with regulations & $<0.001$ & Statistically significant \\
\hline Competitive pressure & .008 & Statistically significant \\
\hline Physical location & .148 & Not statistically \\
\hline significant
\end{tabular}

Other factors influencing the use of cloud computing technology that most participants mentioned (security, privacy, trust, compliance with regulations, compatibility issues and physical location) already exist in the proposed model (Section III). It is interesting to note that security, privacy and trust issues were the big concerns for most organisations participating in this study, and were the main reasons behind their decisions not to adopt cloud services. Also, compliance with regulations is an important factor impacting cloud adoption. One participant stressed that their organisation decided to adopt cloud computing technology 
in limited scale only due to security, privacy issues and legal compliance. The examples below illustrate these issues:

"I think the security issue is the big concern. The company is still hesitant about the security of the data offered by the cloud vendor" (Expert L).

"We didn't use it because it's not secure these days" (Expert Q).

"Trust issues: before adopting, you should know that you will be surrendering all your company's sensitive information to a third-party cloud service provider. This could potentially put your company at great risk. Our data are important. If we are migrating this to a cloud system, the third party will see all the data and everything" (Expert $\mathrm{O})$.

"No rules or regulations govern such direction, and this is one of the reasons behind not adopting cloud service in our organisation. As a result, we need to update our rules and regulations to comply with cloud services. " (Expert I).

Moreover, the experts suggested some other important factors that need to be considered before adopting the cloud, but most of them have already been discussed in the proposed model, such as cost savings, compatibility and availability of cloud service.

In order to increase the precision of this initial study, Cronbach's alpha was used to assess the reliability of the results. Field [20] and Pallant [21] suggested that a value ranging from 0.7 to 0.8 is acceptable. Therefore, the Cronbach's alpha of the interviews with the experts $(0.719)$ is an acceptable value.

\section{CONCLUSION}

To understand the basic concepts of cloud computing, this paper started by providing an overview of this technology. Cloud computing offers several benefits for enterprises such as cost reduction, flexibility, and green IT. But, the idea of migrating an existing system to the cloud is still affects on organisation's decision. Therefore, this research considers how to encourage organisations to adopt cloud computing services and investigates the factors that may influence an organisation's intention to adopt cloud computing. Future work will be focused on validating the proposed model through self-administrated questionnaires, and further results will be published soon.

\section{REFERENCES}

[1] K. Jeffery and B. Neidecker-Lutz, The Future of Cloud Computing Opportunities for European Cloud Computing Beyond, Expert Group Report, Public Version 1.0, 2010.

[2] X. Chen, G. Wills, L. Gilbert, and D. Bacigalupo,"Using cloud for research: a technical review," JISC Final Report, 2010.

[3] R. Buyya, C. S. Yeo, S.Venugopal, J.Broberg, and I. Brandic, "Cloud computing and emerging IT platforms: vision, hype and reality for delivering computing as the $5^{\text {th }}$ utility," Future Gener.Comput. Syst.,vol. 25, pp. 599-616, 2009.
[4] S. Marston, Z. Li, S. Bandyopadhyay, J. Zhang, and A.Ghalsasi, "Cloud computing - the business perspective," Decis. Support Syst., vol. 51, no. 1,pp. 176-189, 2011. doi:10.1016/j.dss.2010.12.006

[5] C. Low, Y. Chen, and M. Wu, "Understanding the determinants of cloud computing adoption," Ind. Manag. Data Syst., vol. 111, no. 7, pp. 1006-1023, 2011, doi:10.1108/02635571111161262.

[6] M. Nkhoma and D. Dang, "Contributing factors of cloud computing adoption: a technology-organisation-environment framework approach,” Int. J. Inform. Syst. Eng., vol. 1, no. 1, 2013, pp. 38-49.

[7] M. Armbrust, A. Fox, R. Griffith, A. D. Joseph, R. H. Katz, A. Konwinski, G. Lee, D. Patterson, A. Rabkin, I. Stoica, and M. Zaharia, "A view of cloud computing," Commun. ACM, vol. 53, Apr. 2010, pp. 50-58.

[8] R. Buyya, R. N. Calheiros, and X. Li, "Autonomic Cloud Computing: Open Challenges and Architectural Elements," Proc. 3rd Int. Conf. on Emerging Applications of Information Technology (EAIT), IEEE Press, 2012, pp. 3-10, doi:10.1109/EAIT.2012.6407847.

[9] I. Foster, Y. Zhao, I. Raicu, and S. Lu, "Cloud Computing and Grid Computing 360-Degree Compared," Grid Computing Environments Workshop (GCE'08), IEEE Press, 2008, pp. 110.

[10] L. G. Tornatzky and M. Fleischer, The Process of Technological Innovation. Lexington, MA: Lexington Books, 1990.

[11] P. Y. K. Chau and K. T. Tam, "Factors affecting the adoption of open systems: an exploratory study," MIS Quarterly,vol. 21, pp. 1-24,1997.

[12] E. M. Rogers, Diffusion of Innovation, 4th ed., New York: Free Press, 1995.

[13] E. M. Rogers, Diffusion of Innovation, 5th ed., New York: The Free Press, 2003.

[14] W. R. Scott, and S. Christensen, The Institutional Construction of Organizations: International and Longitudinal Studies. Thousand Oaks, CA: Sage, 1995.

[15] W. R. Scott, Institutions and Organizations, 2nd ed., Thousand Oaks, CA:Sage, 2001.

[16] B.-Y. Chang, P. H. Hai, D.-W.Seo, J.-H. Lee, and S. H. Yoon, "The Determinant of Adoption in Cloud Computing in Vietnam," Int. Conf. on Comput., Manage. and Telecommun. (ComManTel), IEEE Press, 2013, pp. 407-409, doi:10.1109/ComManTel.2013.6482429

[17] A. Abdollahzadehgan, M. M.Gohary, A.Razak, C. Hussin, and M.Amini, "The Organizational Critical Success Factors for Adopting Cloud Computing in SMEs," J. of Inform. Syst. Res. and Innovation, vol. 4, no. 1, 2013, pp. 67-74.

[18] A. Benlian and T. Hess, "Opportunities and risks of softwareas-a-service: findings from a survey of IT executives," Decis. Support Syst., vol. 52, no. 1,pp. 232-246, 2011.

[19] P. Jaeger, J. Lin, J. Grimes, and S. Simmons, "Where is the cloud? Geography, economics, environment, and jurisdiction in cloud computing," First Monday, vol. 14, no. 5, 2009.

[20] A. Field, Discovering Statistics Using SPSS, 3rd ed., Sage Publications Limited, 2009.

[21] J. Pallant, SPSS survival manual: A step by step guide to data analysis using SPSS, 4th ed., McGraw-Hill Education, 2010. 